\title{
Targeted deep sequencing revealed variants in cell-free DNA of hormone receptor-positive metastatic breast cancer patients
}

\author{
Corinna Keup ${ }^{1}$ (1) $\cdot$ Karim Benyaa ${ }^{2} \cdot$ Siegfried Hauch $^{2} \cdot$ Markus Sprenger-Haussels $^{2} \cdot$ Mitra Tewes $^{3} \cdot$ Pawel Mach $^{1}$. \\ Ann-Kathrin Bittner ${ }^{1} \cdot$ Rainer Kimmig ${ }^{1} \cdot$ Peter Hahn $^{2} \cdot$ Sabine Kasimir-Bauer ${ }^{1}$
}

Received: 4 February 2019 / Revised: 7 June 2019 / Accepted: 11 June 2019 / Published online: 28 June 2019

(c) The Author(s) 2019

\begin{abstract}
Cell-free DNA (cfDNA) is described to mirror intratumoral heterogeneity and gives insight about clonal evolution for improved therapeutic decisions. We sequenced cfDNA of a hormone receptor-positive, HER2-negative metastatic breast cancer (MBC) cohort with a high coverage to examine the prevalence and relevance of any detected variant. cfDNA of 44 $\mathrm{MBC}$ patients was isolated, followed by library construction using a customized targeted DNA panel with integrated unique molecular indices analyzing AKT1, AR, BRCA1, BRCA2, EGFR, ERCC4, ERBB2, ERBB3, ESR1, FGFR1, KRAS, MUC16, PIK3CA, PIK3R1, PTEN, PTGFR, and TGFB1. CfDNA was sequenced on the NextSeq ${ }^{\circledR} 550$ platform (Illumina) and variants were analyzed with Ingenuity Variant Analysis (QIAGEN). We evaluated cfDNA variants in 40 of the 44 hormone receptor-positive and HER2-negative patients with a high mean coverage of 22,000×, resulting in $M U C 16, B R C A 2, E R B B 3$, and $A R$ variant calling in $>90 \%$ of the patients. $47 \%$ of all $A R$ variants were pathogenic and at least one pathogenic or likely pathogenic variant was detected in each patient. A specific $B R C A l$ variant and $>3.5$ pathogenic variants significantly associated with a reduced survival after diagnosis of metastasis. Longitudinal monitoring revealed an increase of pathogenic and likely pathogenic PIK3CA and ESRI variant allele frequency under everolimus and exemestane, 8 months before proof of therapy failure by visual staging in one exemplary case. The identification of new variants with high prevalence, prognostic value, and dynamics under treatment by deep sequencing of cfDNA might empower sensitive monitoring and personalized therapeutic decisions.
\end{abstract}

Keywords Metastatic breast cancer $\cdot$ Liquid biopsy $\cdot$ Therapy stratification $\cdot$ Next generation sequencing $\cdot$ NGS . Circulating tumor DNA $\cdot \operatorname{ctDNA} \cdot \operatorname{ccfDNA} \cdot$ Mutation $\cdot \mathrm{SNP} \cdot$ Unique molecular indices

\begin{tabular}{ll}
\multicolumn{2}{l}{ Abbreviations } \\
AF & Allele frequency \\
BC & Breast cancer \\
cfDNA & Cell-free DNA
\end{tabular}

Peter Hahn and Sabine Kasimir-Bauer contributed equally to this work.

Electronic supplementary material The online version of this article (https://doi.org/10.1007/s00018-019-03189-z) contains supplementary material, which is available to authorized users.

Corinna Keup

Corinna.Keup@uk-essen.de

1 Department of Gynecology and Obstetrics, University Hospital of Essen, Essen, Germany

2 QIAGEN GmbH, Hilden, Germany

3 Department of Medical Oncology, University Hospital of Essen, Essen, Germany

$\begin{array}{ll}\text { ctDNA } & \text { Cell-free tumor DNA } \\ \text { ER } & \text { Estrogen receptor } \\ \text { HR } & \text { Hormone receptor } \\ \text { IQR } & \text { Interquartile range } \\ \text { IVA } & \text { Ingenuity variant analysis } \\ \text { NGS } & \text { Next generation sequencing } \\ \text { MBC } & \text { Metastatic breast cancer } \\ \text { PR } & \text { Progesterone receptor } \\ \text { UMI } & \text { Unique molecular indice }\end{array}$

List of genes

AKT1 AKT serine/threonine kinase 1

$A R \quad$ Androgen receptor

BRCA1 BRCA1 DNA repair associated

BRCA2 BRCA2 DNA repair associated

EGFR Epidermal growth factor receptor

ERCC4 ERCC excision repair 4, endonuclease catalytic subunit 


$\begin{array}{ll}\text { ERBB2 } & \begin{array}{l}\text { Erb-b2 receptor tyrosine kinase 2: encoding for } \\ \text { HER2 }\end{array} \\ \text { ERBB3 } & \text { Erb-b2 receptor tyrosine kinase 3: encoding for } \\ & \text { HER3 } \\ \text { ESR1 } & \text { Estrogen receptor 1 } \\ \text { FGFR1 } & \text { Fibroblast growth factor receptor 1 } \\ \text { KRAS } & \text { KRAS proto-oncogene, GTPase } \\ \text { MUC16 } & \text { Mucin 16, cell-surface associated } \\ \text { PIK3CA } & \begin{array}{l}\text { Phosphatidylinositol-4,5-bisphosphate 3-kinase } \\ \text { catalytic subunit alpha }\end{array} \\ \text { PIK3R1 } & \begin{array}{l}\text { Phosphoinositide-3-kinase regulatory subunit 1 } \\ \text { PTEN }\end{array} \\ \text { Phosphatase and tensin homolog } \\ \text { PTGFR } & \text { Prostaglandin F receptor } \\ \text { TGFB1 } & \text { Transforming growth factor beta 1 }\end{array}$

\section{Introduction}

Currently, liquid biopsies appear as promising tools for individualized treatment decisions and real-time monitoring strategies in oncology including breast cancer (BC). Cell-free DNA (cfDNA), specifically cell-free tumor DNA (ctDNA), defined by the presence of variants [1], represents tumor heterogeneity, because ctDNA can be released by tumor cells in the primary tissue, in metastases, and in circulation. Moreover, ctDNA harbors newly acquired variants selected by and/or expanding under therapy [2], therefore, mirroring clonal evolution. In contrast, tissue biopsies only depict a spatially and temporally limited snapshot and are often not feasible. CfDNA, reflecting the characteristics of the metastases better than those of the primary tumor [3], could serve as easily assessable alternative for biopsies of metastases.

The relevance of cfDNA in $\mathrm{BC}$ has already been reported for diagnostic, prognostic, predictive, and monitoring approaches [4]. The prognostic value of ctDNA is undisputable in BC, since high levels of ctDNA were associated with poor overall survival (OS) [5]. ESR1 variants were specifically correlated with shorter duration of endocrine treatment effectiveness in metastatic BC (MBC) [6] and PIK3CA variants in exon 20 , significantly associated with poor prognosis [7]. Regarding the predictive value, patients with ESRI variants were demonstrated to benefit from fulvestrant rather than from exemestane, compared to patients without this somatic alteration [8]. In the BELLE-2 study, the addition of a pan-PI3K inhibitor resulted in an improved progressionfree survival only in patients with $P I K 3 C A$-altered cfDNA [9]. For disease monitoring, cfDNA concentration was reported to indicate impending relapse of primary BC earlier than any other imaging or blood-based strategy [10] and could predate treatment response changes $[11,12]$.

Detection methods for cfDNA variants are as diverse as their advantages and disadvantages $[1,13]$. The rapid development of next generation sequencing (NGS) methods supported the unbiased variant analysis in the cfDNA research field. Due to the low input requirements, targeted PCR-based library techniques are preferred over hybridcapture methods [14]. However, PCR-based techniques are prone to artifacts due to the error rate of polymerases and, thus, display a decreased sensitivity of around 1\% [1]. Modern molecular barcoding techniques handle this problem and bioinformatically remove PCR artifacts to detect true positive variants at allele frequencies (AFs) of down to $0.1 \%$ [4, 10].

We here (a) established a targeted PCR-based NGS approach with integrated unique molecular indices (UMIs) and extremely high coverage to analyze cfDNA variants from hormone receptor (HR)-positive and HER2-negative MBC patients in detail and (b) compared results with and without UMIs, (c) examined variants in MUC16, BRCA2, ERBB3, AR, EGFR, PTEN, ERBB2, PIK3CA, BRCA1, ESR1, PTGFR, TGFB1, AKT1, FGFR1, ERCC4, PIK3R1, and $K R A S$, and (d) explored the development of likely pathogenic and pathogenic cfDNA variants of one particular HR+ HER2- patient from the time point of primary tumor biopsy until death to get insight about the value of cfDNA variants for treatment decision making and monitoring.

\section{Subjects and methods}

\section{Patient population characteristics and eligibility criteria}

The study was carried out at the Department of Gynecology and Obstetrics, in collaboration with the Department of Medical Oncology (for specimen recruitment), both at the University Hospital Essen, Germany and in collaboration with QIAGEN GmbH, Hilden, Germany (for library preparation and sequencing analysis). The eligibility criteria have been previously published [15]. Written informed consent was obtained from all participants at enrollment and specimens were collected using protocols approved by the institutional review board (12-5265-BO). In total, cfDNA from 44 MBC patients was studied between March 2013 and August 2017. MBC patients with estrogen (ER) and/or progesterone (PR) receptor-positive primary tumors without $E R B B 2$ overamplification were enrolled. Patients with ER-positive and/or PR-positive and HER2-negative metastases (if multiple pathology reports of metastases were available, we used the report of metastasis biopsy that was taken closest to the date of blood draw for this study) had also been included if their ER, PR and HER2 status in the primary tumor was unknown. Patient characteristics of the 40/44 cases finally 
fulfilling the stringent sequencing quality parameter and thus used for variant analysis are listed in Online Resource 1.

\section{Sampling of blood, processing of plasma, and isolation of cfDNA}

$9 \mathrm{ml}$ EDTA blood was collected in S-Monovettes ${ }^{\circledR}$ (Sarstedt, Germany), stored at $4{ }^{\circ} \mathrm{C}$, and centrifuged within $4 \mathrm{~h}$ after withdrawal at $1841 \times \mathrm{g}$ for $8 \mathrm{~min}$. Plasma was stored at $-80{ }^{\circ} \mathrm{C}$. Thawed plasma was centrifuged at $16,000 \times g$ for $10 \mathrm{~min}$ at $4{ }^{\circ} \mathrm{C}$ and passed through a $0.8 \mu \mathrm{m}$ pore size syringe filter (Sartorius, Germany). cfDNA was isolated from 1.8 to $5.4 \mathrm{ml}$ (preferably $4 \mathrm{ml}$ ) plasma by affinity-based binding to magnetic beads according to the manufacturer's instructions (QIAamp MinElute ccfDNA Kit, QIAGEN, Germany) and was eluted in $22 \mu \mathrm{l}$ ultraclean water.

\section{cfDNA quantification}

Diluted cfDNA (1:2-1:100) was applied to the Agilent Chip High Sensitivity DNA (Santa Clara, US). Concentrations of fragments with a length between 100 and $700 \mathrm{bp}$ were added up using the 2100 expert software B02.08 to calculate the cfDNA yield.

\section{Library construction}

The library was constructed with the QIAseq Targeted DNA Panel Kit (QIAGEN) and according the manufacturer's instructions. The input amount preferred for library preparation was in the range of 30-60 ng, but cfDNA samples with lower input were also included in the library preparation. Because the concentration of some cfDNA eluates was critically low, we used an input volume of $20 \mu \mathrm{l}$ for all libraries, instead of $16.75 \mu \mathrm{l}$ as described in the handbook. Thus, the reagent volumes had to be adjusted as described and published at protocols.io [16]. Briefly, end-repair and a-addition was performed, while the enzymatic fragmentation was inhibited. In the next step, the UMI and samplespecific adapter were ligated to the fragments $[14,17]$. DNA was purified and size selected by magnetic beads. The targeted enrichment was performed with customized QIAGEN QIAseq Targeted DNA Panel primer designed to amplify all coding regions of $A K T 1, \mathrm{AR}, B R C A 1, B R C A 2, E G F R$, ERBB2, ERBB3, ERCC4, ESR1, KRAS, FGFR1, MUC16, PIK3CA, PIK3R1, PTEN, PTGFR, and TGFBI and exhibited high specificity and uniformity (Online Resource 2). The universal PCR amplification and integrated additional sample-specific adapter ligation was followed by a magnetic bead cleanup and the final targeted enriched cfDNA library was eluted.

\section{Sequencing}

Libraries were quantified as published at protocols.io [16] by qPCR and the quality was checked by Agilent Chip High Sensitivity DNA. Libraries were diluted to $2 \mathrm{nM}$ (MiSeq, Illumina) or $4 \mathrm{nM}$ (NextSeq, Illumina). Libraries with a lower yield were excluded. All pooled libraries were analyzed by in total four runs paired-end sequencing on two different Illumina sequencers using a custom sequencing primer (QIAseq A Read1 Primer). One run was performed on the Illumina MiSeq Sequencer with the MiSeq Reagent Kit v2, $2 \times 150$ bp reads with some samples for the comparison \pm UMIs. All other libraries were sequenced on the Illumina NextSeq Sequencer with a NextSeq 550 System High-Output Kit, 2x150 bp reads.

\section{Data analysis/bioinformatical analysis}

Data were initially analyzed using the QIAGEN GeneGlobe Data Analysis Center. Sufficient sequencing quality of all samples for clinical interpretation had been guaranteed by exclusion of libraries with less than 5 million read fragments, an UMI coverage lower than 400 and if less than $95 \%$ of the target region was covered with at least $5 \%$ of the mean UMI coverage, as described [16, 18]. The QIAGEN Biomedical Genomics Workbench and the Ingenuity Variant Analysis plugin (IVA; QIAGEN) were used for further annotation, scoring, filtering (described previously $[16,18]$ ) and interpretation of variants detected in the UMI-based analysis. Called variants were separately annotated for the different transcript isoforms. Original raw sequencing data are available at the European Nucleotide Archive with the study accession number PRJEB29032 and sequencing quality parameter are listed in Online Resource 3.

\section{Statistical analysis}

Cut-off values for subsequent survival analysis were determined by empirical ROC analysis and a maximized Youden's index. Kaplan-Meier survival analysis was interpreted by log-rank (Mantel-Cox) test for all variants with a frequency of $15-85 \%$ in the cohort and for the parameters: number of variants detected, number of likely pathogenic and pathogenic variants detected, and mean AF of all detected variants. Significant values were indicated as asterisks ( $* \hat{=} p$ value $<0.05)$. Statistical analysis was performed by SPSS, version 11.5 (SPSS Inc.). 


\section{Results}

\section{Integration of UMIs in the library and its consequences on called PIK3CA hotspot variants}

To demonstrate the differences in results with and without use of UMIs, two different bioinformatical strategies were compared in samples that presented PIK3CA hotspot variants (P536S, E545K, and H1047R) with an AF $<3 \%$ in the workflow without UMI consolidation $(n=10)$. After integration of UMIs in the bioinformatical analysis $50 \%$ of the called variants without UMI consolidation were verified as true positives (Fig. 1). The PIK3CA hotspot variant with the lowest $\mathrm{AF}(0.72 \%)$ verified as true positive by UMIs was detected in a sample with a high cfDNA input (60 ng) and deep sequencing qualities (mean coverage: 22,000x, UMI coverage: 6351). In contrast, a sample with low cfDNA input (7 ng) sequenced on the MiSeq (mean coverage: 3,000x, UMI coverage: 230) failed in verification of $P I K 3 C A$ H1047R detected without UMI consolidation (AF: 1.56\%). In conclusion, high cfDNA input amount and corresponding good sequencing qualities enable verification of true positive variants by UMIs. Consequently, results described in the following sections were all confirmed by consolidation of UMIs.

\section{Insight into the characteristics of variants found in the HR+ HER2- MBC cohort}

For the evaluation of clinically relevant insights disclosed in cfDNA, cfDNA was isolated of a histologically confirmed cohort of 44 HR+ HER2- MBCs. After sequencing, data of 4/44 samples were excluded due to application of stringent exclusion criteria for sequencing quality parameters.
Sequencing quality parameter of the remaining 40 samples and corresponding patient characteristics are listed in the Online Resources 1 and 3. Importantly, a mean of 18 million fragment reads was analyzed in each sample with a mean coverage of $21,600 \times$ and a mean UMI coverage of 2668 indicating deep sequencing quality of all samples. Variants with a prevalence of $>3 \%$ in the normal reference population were excluded unless the variant was already known to be a pathogenic common variant.

In total, 3415 variants were found in the cfDNA of all patients sequenced using a targeted PCR-based NGS approach with integrated UMIs analyzing MUC16, BRCA2, ERBB3, AR, EGFR, PTEN, ERBB2, PIK3CA, BRCA1, ESR1, PTGFR, TGFB1, AKT1, FGFR1, ERCC4, PIK3R1, and $K R A S$. Of these detected variants, $6.5 \% / 7.2 \%$ were identified as pathogenic/likely pathogenic by IVA, while $83.4 \%$ variants were of uncertain significance (Fig. 2a). Of all variants, $74 \%$ were missense variants (Fig. 2b) and 93\% were single nucleotide polymorphisms (Fig. 2c).

Of all 3415 variants, $31 \%$ were found in the MUC16 gene that thus was the gene with the most variants detected of all 17 tested genes (Fig. 2d). ERBB2 was the gene with the second highest number of variants detected (11.3\%). Interestingly, half of the detected variants (49\%) showed an $\mathrm{AF}$ of between 1 and $5 \%$ and $43 \%$ of all variants were detected with an $\mathrm{AF}$ of $<1 \%$ (Fig. 2e).

Of all variants, $465 / 3415$ variants were described to be pathogenic or likely pathogenic using IVA. Strikingly, $47 \%$ of all pathogenic or likely pathogenic variants were found in the $A R$ gene (Fig. 2f); 5.4\%/4.3\% of all pathogenic and likely pathogenic variants were detected in the PIK3CA/ESR1 gene. The fraction of variants with an AF of $20-50 \%$ increased by filtering only pathogenic and likely pathogenic variants from all variants $(4.9 \%$ compared to $1.3 \%$; Fig. 2e, g).

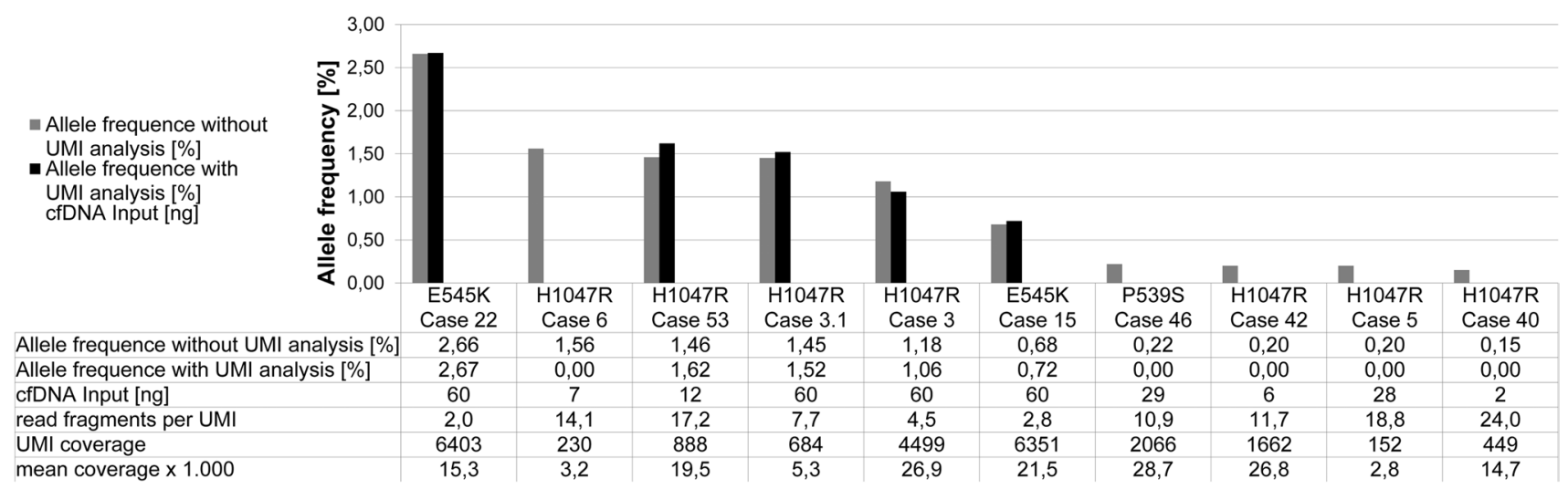

Fig. 1 Comparison of called PIK3CA hotspot variants with (black) and without (grey) unique molecular indices (UMIs). Only cases with variant $\mathrm{AFs}<3 \%$ are examined. Detection of low fraction PIK3CA hotspot variants with UMIs is dependent on high cfDNA input, as well as on a high UMI coverage and a small ratio of read fragments per UMI 

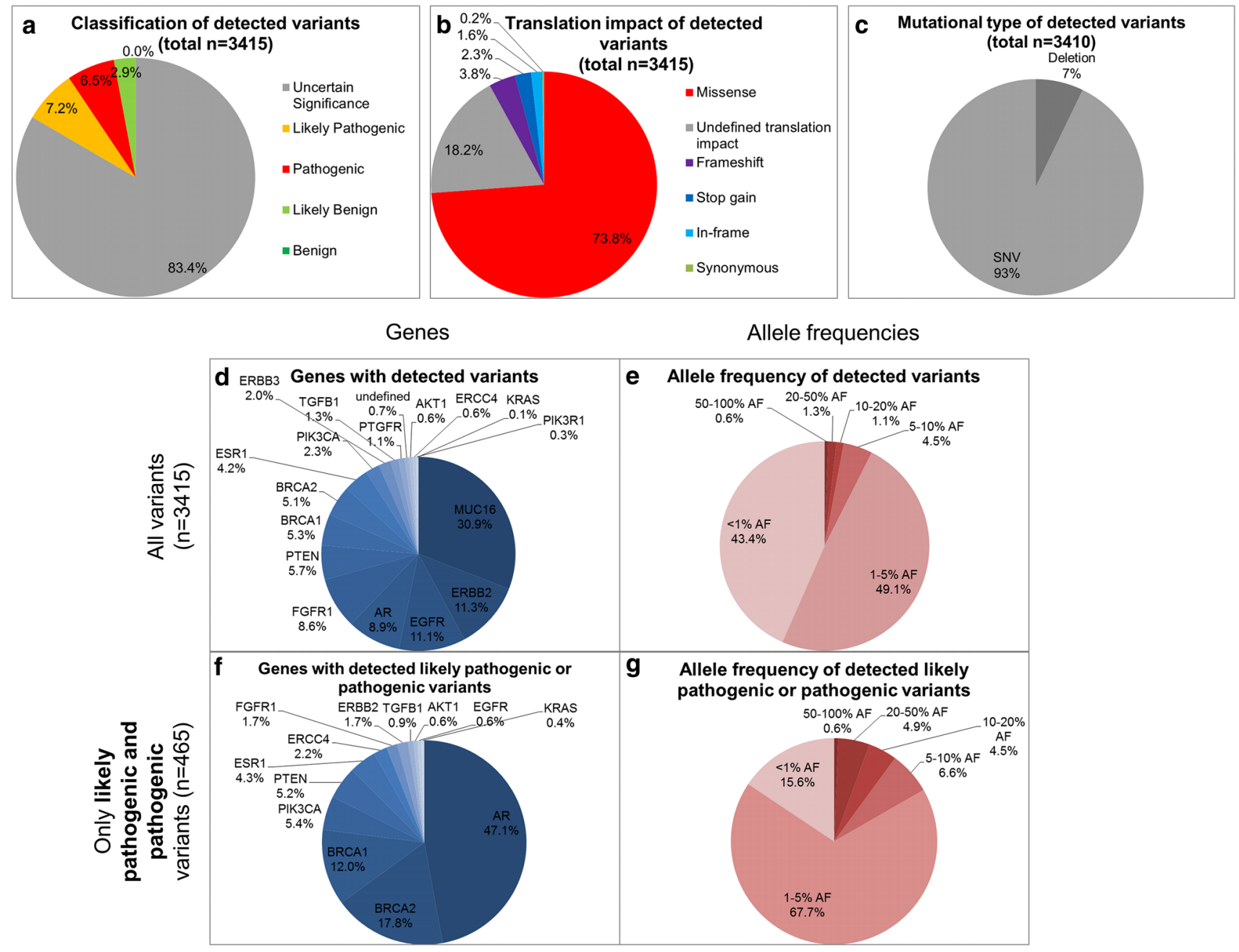

Fig. 2 Characteristics of called variants in cfDNA in $H R+H E R 2-M B C$ patients $(n=40)$. a Classification of variants according to their known impact (benign, likely benign, uncertain significance, likely pathogenic and pathogenic) [26] done by IVA. Of all detected variants $(n=3415), 14 \%$ are likely pathogenic or pathogenic. b Translation impact of detected variants. Three quarter of all variants were missense variants. c Differentiation into mutational types. Only $n=5$ were multi nucleotide polymorphisms or insertions, while
93\% were single nucleotide polymorphisms. d Distribution of variants regarding their gene location. Most variants were found in the MUC16 gene. e AFs of all variants. $90 \%$ of all variants displayed $\mathrm{AFs}<5 \%$. f Distribution of all pathogenic or likely pathogenic variants $(n=465)$ regarding their gene location. Most pathogenic or likely pathogenic variants found in the $A R$ gene. $\mathbf{g} A F s$ of all pathogenic and likely pathogenic variants. $68 \%$ presented an AF between 1 and $5 \%$

Twelve MUC16 variants, six PTEN variants, four $E R B B 2, A R$, and $E G F R$ variants, and two $B R C A 2$ variants were detected with a prevalence of $>50 \%$ (Online Resource File 4). The two most common variants in the cohort were MUC16 c.39020T > G p.V13007G, detected in $38 / 40$ patients (95\% prevalence, mean $\mathrm{AF} 3.2 \%$ ) and MUC16 c.17434_17436delACT p.T5812del identified in 34/40 samples (85\%; mean AF 0.8\%) (Online Resource $4)$.

and $A R$ was $>90 \%$ (Fig. 3). Except KRAS and PIK3RI, all tested genes displayed variants in at least $30 \%$ of all patients (for example, PIK3CA 73\% and ESR1 55\%). 


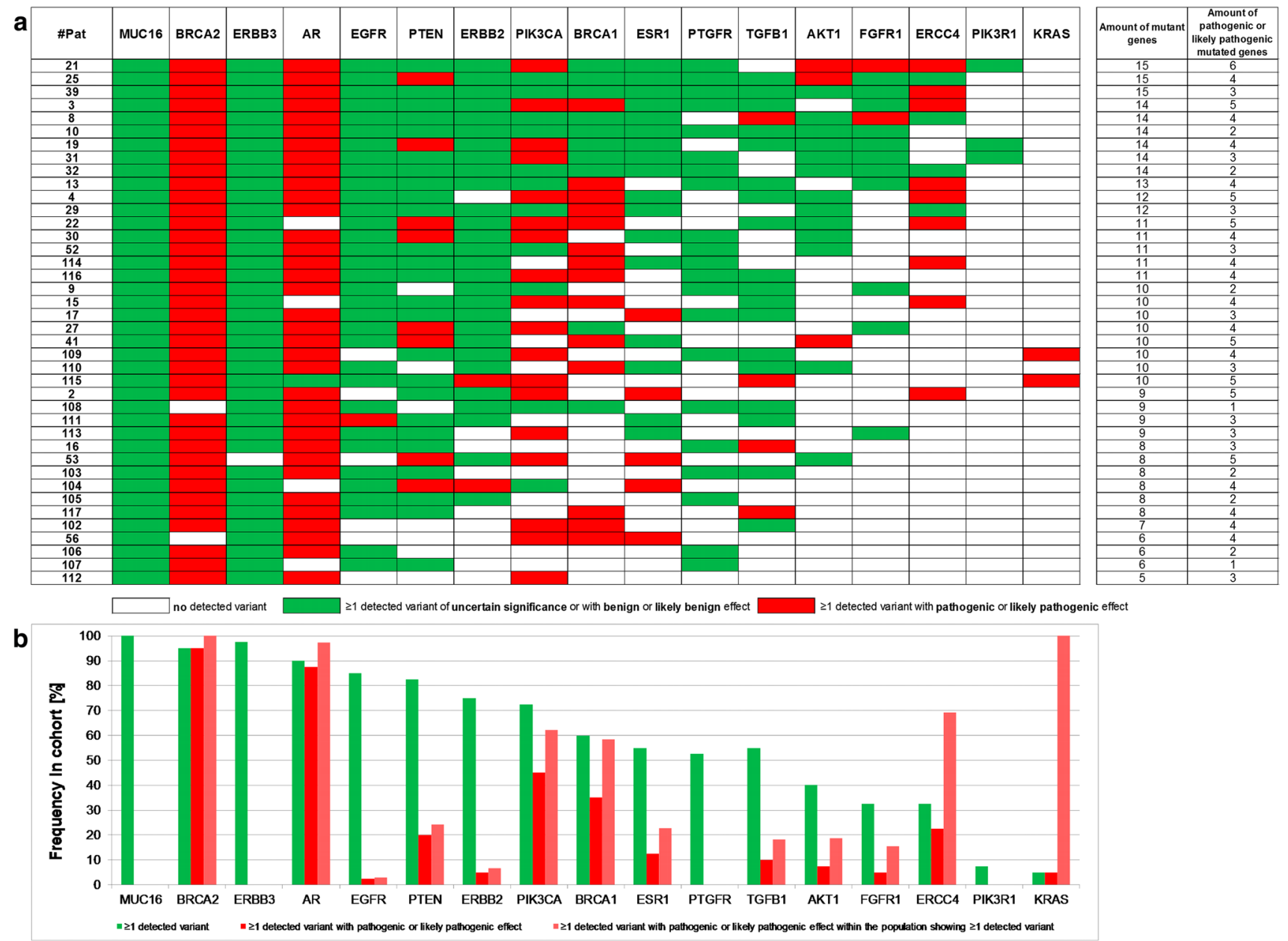

Fig. 3 Prevalence of altered genes. a Heatmap of mutated genes in each of the 40 patients. Genes with at least one variant are illustrated in color. Genes with at least one likely pathogenic or pathogenic variant are presented in red. Patients were sorted according to the amount of altered genes. Each patient displayed at least one pathogenic or likely pathogenic variant in one of the 17 tested genes and showed at least one MUC16 variant. b Prevalence of altered genes in percent- age. Frequency of altered genes (green), frequency of pathogenic or likely pathogenic altered genes (red) and frequency of pathogenic or likely pathogenic alterations within the cohort showing any variant in this gene (pink) are depicted. MUC16, BRCA2, ERBB3 and $A R$ alterations showed a prevalence of $>90 \%$. BRCA2 and $A R$ were the most frequently altered genes with pathogenic or likely pathogenic effect

exonic variant with likely pathogenic impact and greatest prevalence was $A R \mathrm{H} 382 \mathrm{P}(80 \%)$.

\section{Prognostic value of cfDNA variants}

The median survival time in the HR+HER2- MBC cohort ( $n=40$; Online Resource 1) after diagnosis of metastasis was 46 months with an interquartile range (IQR) of 31 months. At the examination time point for the prognostic value of the detected variants in the cohort by Kaplan-Meier analysis, 29 patients were deceased, while 11 were still alive (Online Resource 1). 
a Number of pathogenic or likely pathogenic variants

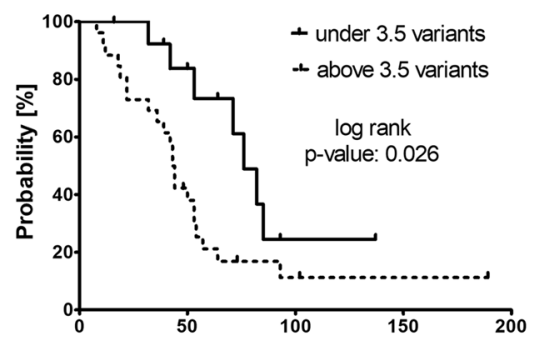

survival after diagnosis of metastasis [months] b

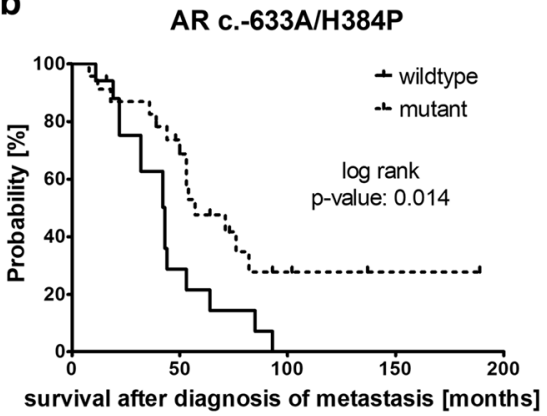

MUC16 R10996S

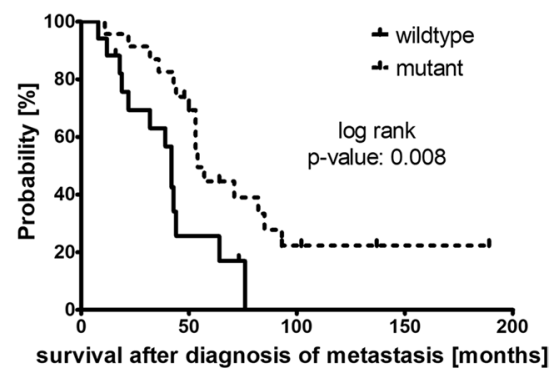

BRCA1 c.787+1174delA/ K607fs*47/K607fs*74

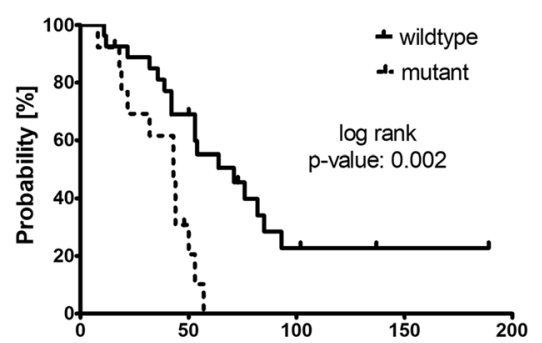

survival after diagnosis of metastasis [months]
ERBB2

P582L/P597L/P612L

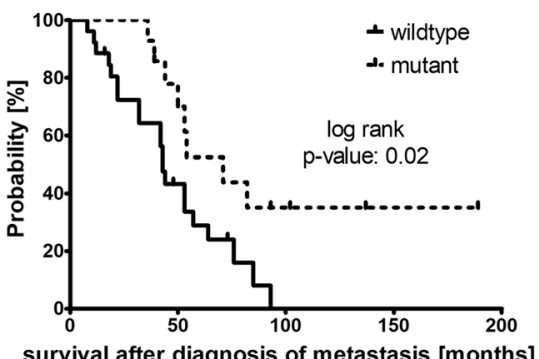

MUC16 S836R

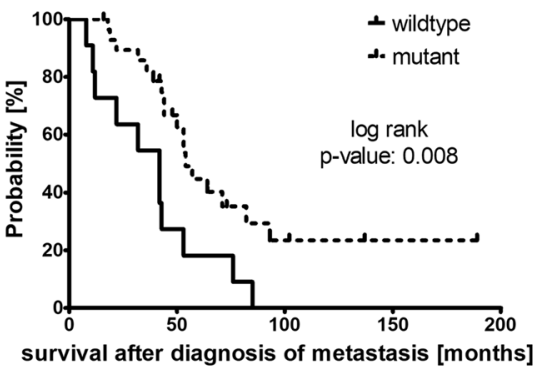

MUC16 P835A

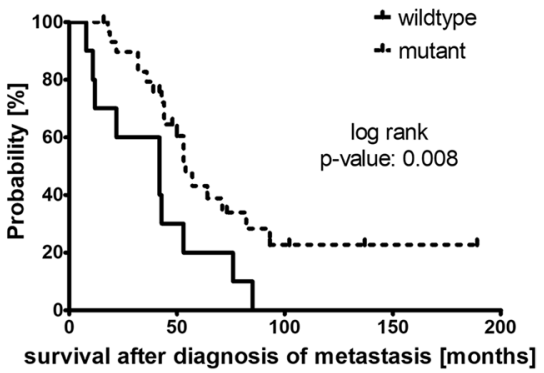

Fig. 4 Survival analysis by Kaplan-Meier curves and log-rank (Mantel-Cox) test. a Kaplan-Meier curves illustrating parameter significantly correlated with reduced survival after diagnosis of metastasis. Cut-off values were calculated by ROC analysis and Youdens' index. Patients with $>3.5$ pathogenic and likely pathogenic variants or with $B R C A 1$ variant c.787+1174delA/K607fs*47/K607fs*74 (different

The number of likely pathogenic or pathogenic variants was significantly correlated with decreased survival time after diagnosis of metastasis (threshold identified by ROC analysis and Youden's index: $3.5 ; p$ value of log-rank test for Kaplan-Meier curve: 0.026; Fig. 4a and Online Resource 6).

Of all tested individual variants with a prevalence between 15 and $85 \%(n=186), 16$ variants (if the variants annotated to the different isoforms are counted separately) displayed a significant correlation $[p$ value $<0.05$ by logrank (Mantel-Cox) test] with survival after diagnosis annotations according to different $B R C A 1$ isoforms) displayed a significant correlation with worse survival after diagnosis of metastasis. b Single variants significantly correlated with increased survival after diagnosis of metastasis. One single variant of $E R B B 2$ and $A R$ and three MUC16 variants were observed to increase the survival after diagnosis of metastasis highly significantly

of metastasis (Online Resource 6). The presence of the $B R C A 1$ c. $787+1174$ delA/K607fs*47/K607fs*74 variant (annotated to different BRCAl isoforms) significantly correlated with worse survival after diagnosis of metastasis (Fig. 4a), while one $A R$ variant, three MUC16 variants, and one $E R B B 2$ variant were significantly associated with increased survival after diagnosis of metastasis (Fig. 4b). In addition, the correlation of specific variants with the interval between blood draw and death and the progression-free survival under the therapy given at/after 
Fig. 5 Longitudinal monitoring of cfDNA variants with pathogenic/likely pathogenic impact in one HR+ HER2 patient. CfDNA was isolated and sequenced at six time points across treatment in a period of 3 years. Cumulative altered fragment counts of all detected pathogenic and likely pathogenic variants are illustrated. As not to sum allele frequencies, cumulative altered fragment counts are displayed. In general, however, it should be considered that wildtype fragment counts were stable across time points and variant locations, while altered fragment counts exhibited dramatic changes. Variants located in the same gene are shown in the same color but different shade. Applied therapies as well as staging results (by visual staging and RECIST) are depicted as well

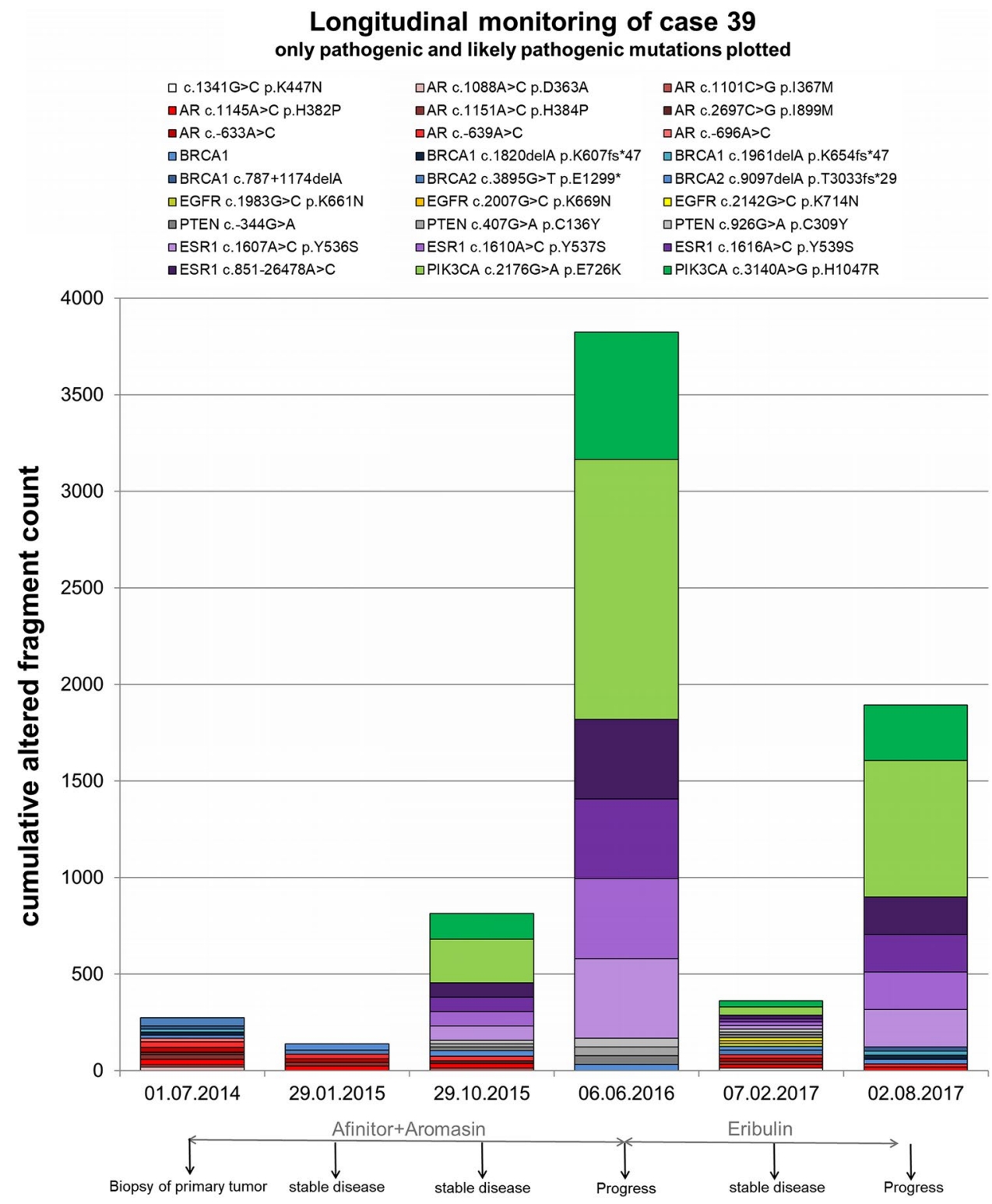

Longitudinal monitoring of case 39 $\square A R$ c. $1101 C>G$ p. $1367 \mathrm{M}$ $A R$ c. $-696 \mathrm{~A}>\mathrm{C}$ the blood draw was evaluated in all variants with a prevalence between 15 and 85\% ( $n=186$; Online Resource 6).

\section{Longitudinal monitoring across treatment}

Here, we describe the development of pathogenic and likely pathogenic cfDNA variants and their AFs in one MBC patient with ER+ PR- HER2- primary tumor at six time points in a period of 3 years. She was diagnosed with multicentric ductal $\mathrm{BC}$ at grade two and osseous metastases in March 2014 at the age of 61 years and died with cutaneous, pulmonary, osseous, pleural, and hepatic metastases in October 2017.

In July 2014, the primary tumor was biopsied and the first plasma sample was taken, showing no pathogenic or likely pathogenic variants in the PIK3CA or ESRl gene (Fig. 5).
Afterwards, the patient received everolimus and exemestane which resulted in a stable disease for nearly 2 years until disease progression in June 2016. However, the cfDNA variant development assessed retrospectively in this study showed the appearance and even increase in AF of PIK3CA E726K (Oct 2015 9.8\%; June 2016 38.3\%), PIK3CA H1047R (Oct 2015 11.4\%; June $201639.0 \%$ ), ESRl Y536S, ESRl Y537S, ESRl 539S and one ESR1 intronic variant (AF of all ESRI variants: Oct 2015 4.1\%; June 2016 14.0\%) in this time frame. Hence, we identified these pathogenic PIK3CA and ESR I variants under mTOR and aromatase inhibitor therapy at the staging time point, 8 months earlier than the visual staging time point evaluating a progressive disease. Treatment was then changed to eribulin for about a year until disease progression. Therapy change first induced decreased PIK3CA and ESRI variant AFs. At the progressive time point under eribulin, however, the 
PIK3CA and ESR 1 variant AFs were dramatically increased again (PIK3CA E726K 2.9\%-24.6\% from Feb 2017 to Aug 2017; PIK3CA H1047R 3.3\%-26.0\% and all ESRI variants $1.3 \%-9.1 \%)$. All in all, two PIK3CA variants and four ESRI variants appeared during everolimus and exemestane therapy and were prominent at both progressive time points within the observed period with $>9 \%$ AF.

\section{Discussion}

Deep sequencing of variants in all coding regions of MUC16, BRCA2, ERBB3, AR, EGFR, PTEN, ERBB2, PIK3CA, BRCA1, ESR1, PTGFR, TGFB1, AKT1, FGFR1, ERCC4, PIK3R1, and KRAS in cfDNA samples of 40 HR+HER2- MBC patients revealed that each patient displayed at least one pathogenic or likely pathogenic variant and the highest frequency of likely pathogenic or pathogenic alterations occurred in the $A R$ gene. The prevalence of $M U C 16, B R C A 2, E R B B 3$, and $A R$ variants was $>90 \%$ in this cohort. We here identified single variants with significant correlation with increased survival after diagnosis of metastasis. On the other hand, a BRCA1 variant as well as a number of pathogenic or likely pathogenic variants of greater 3.5 were significantly associated with worse survival after diagnosis of metastasis. The longitudinal monitoring of cfDNA variant evolution of one HR+ HER2- patient revealed the prominence of PIK3CA and $E S R I$ variants before staging the disease to be progressive under everolimus and exemestane.

\section{Implications of input amount, UMI integration, high coverage, and IVA filter}

The required blood volume of $9 \mathrm{ml}$ is comparable to the obligatory blood volume for other liquid biopsy tests used in clinical practice already (such as Guardant $360^{\circledR}$ ctDNA [19] or OncoBEAM ${ }^{\mathrm{TM}}$-RAS-Test) yielding in minimally $4 \mathrm{ml}$ plasma. The high plasma input of $4 \mathrm{ml}$ enabled isolation of cfDNA amounts usable for reliable targeted PCR-based cfDNA library preparation (library yield $>4 \mathrm{nM}$ ). The integration of UMIs was exemplified for identification of PIK3CA hotspot variants in this study and guarantees the identification of only true positive variants, but requires high cfDNA input and deep sequencing qualities. Exactly those deep sequencing qualities were met in all 40 cases used for variant analysis, since 18 million read fragments were sequenced on average in each sample, the mean coverage was $22,000 \times$, and the UMI coverage was 6351 . This high coverage enabled calling variants with low AFs and resulted in the identification of a high prevalence of variants, which has rarely been described before [20-22]. Many studies [2, 23-25] that described a low frequency of variants were conducted with a lack of high coverage, missing descriptions of the input amount or sequencing of hotspots rather than all exonic regions of a gene. We here ensure specificity and relevance of called variants by usage of UMIs and stringent IVA filter settings [16] and variant prevalence detected by analysis of tissue or liquid biopsy should be differentiated. Among other settings, only predicted deleterious variants were called and variants with a prevalence of $>3 \%$ in the healthy reference population (Allele Frequency Community (gnomAD\&CGI), 1000 Genomes Project, ExAC and NHLBI ESP exomes) were excluded. Classification of the variants was conducted according to the guidelines of the association for molecular pathology [26]. Although efforts ensuring a high sensitivity and specificity in variant calling were undertaken, a verification of variants by independent methods in the future would be desirable, which was not conducted here due to the limited cfDNA amount available and the plenty of variant locations to be verified. The detected sequence alterations were separately listed with different variant nomenclature when different isoforms were concerned. This can be one reason for the large number of reported variants. Although we did not compare the data against cfDNA of healthy donors that underwent the same workflow, we excluded variants with a high prevalence in the reference populations. Matched germline samples were not available, thus, we do not claim the described variants to be somatic per se, but low AFs might indicate the variants to be somatic rather than germline and sequencing of matched germline samples has been initiated for future studies.

\section{MUC16 variants}

MUC16 variants were reported to be frequently found in most cancer types $[27,28]$ which is in line with the high prevalence of $M U C 16$ variants in our patient cohort. However, due to the fact that MUC16 consists of $\sim 22,000$ amino acids, the variant frequency corrected by sequence length and mutational heterogeneity resulted in exclusion of MUC16 as one of the most altered genes in most cancer types, including BC [28, 29]. MUC16 variants were found to induce MUC16 overexpression in BC and thus caused increased cancer growth and migration as well as decreased sensitivity to cisplatin [30]. The presented new highly significant association of three MUC16 variants (R10996S, S836R, P835A) with increased survival after diagnosis of metastasis challenges the knowledge about the negative effect of MUC16 variants in BC. In accordance with the statement of decreased sensitivity to cisplatin [30], preliminary data of our cohort showed 
that the mean number of $M U C 16$ variants was reduced in the cohort that had received platinum-based therapy sometime before blood draw $(n=4)$ compared to those patients who had never received any platinum-based therapy ( $n=34)$ (15 versus 28 MUC16 variants), but no significant reduction in survival time comparing both cohorts (107 months versus 117 months) was found (data not shown). A cluster analysis of all MUC16 variants further separated the patients into two distinct groups, but these data are too preliminary to draw any clinical conclusions.

\section{Nuclear steroid hormone receptor variants}

ESRI variants were mostly found in ER+ patients and were induced by endocrine treatment $[2,31]$. ESRl variants in cfDNA of MBC patients were reported to have a prevalence of $25.3 \%$ (PALOMA3), $28.8 \%$ (BOLERO-2) and $39.1 \%$ (SoFEA) [6]. The 55\% ESRI variant prevalence in the presented study may be explained by the inclusion of a majority of $\mathrm{HR}+$ cases in late treatment lines. In this regard, it is to mention that $62 \%$ of all included patients received two or more different endocrine treatment regimens before blood draw including non-steroid aromatase inhibitors, steroid aromatase inhibitors, selective estrogen receptor modulators, or selective estrogen receptor degraders (Online Resource 1).

Longitudinal cfDNA monitoring in one of the patients identified the appearance and increase in AF of ESRI Y536S, ESR1 Y537S, and ESR1 Y539S which might be one potential mechanism of resistance causing progressive disease after 2 years of endocrine treatment. These three variants are located at positions encoding for the ligandbinding domain of ER and cause resistance to aromatase inhibitor therapy and, thus, were already described to be predictive [2, 8, 32-34].

AR expression on the mRNA- and protein level was studied in $\mathrm{BC}$ cases after realization of a complex interplay of androgens and estrogens [35]. However, to the best of our knowledge, we are one of the first studying the genomic alterations in the $A R$ gene in $\mathrm{BC}$ in more detail and strikingly found that $88 \%$ of all patients displayed likely pathogenic and pathogenic (defined by guidelines of the association for molecular pathology [26]) $A R$ variants. Almost half of all likely pathogenic and likely pathogenic variants found in all tested 17 genes were localized in the $A R$ gene, as previously described [18]. Such high prevalence of $A R$ variants should be further studied to elucidate the functional influence of $A R$ variants on the AR expression, subsequently, on AR-dependent pathways and, finally, on BC pathogenesis. DNA regions encoding for glutamine repeats, also called polyQ stretches, are frequently found in the $A R$ gene [36] and were described to be of high functional relevance [36]. However, variant calling in polyQ stretches was found to be challenging [37]. $A R$ splice variants were already detected in $\mathrm{BC}$ patients [38] and $A R-V 7$ was reported to cause resistance to anti-AR therapies in prostate cancer [39]. Consequently, the functional analysis of $A R$ variants might indicate new treatment options for $\mathrm{BC}$ patients with $A R$ variants in the future.

\section{PIK3CA variants}

PIK3CA variants showed a high prevalence in HR+ HER2- BCs (45\%/29\% of luminal A/B BC patients) [40]. Here, we detected PIK3CA variants in $73 \%$ of HR + HER2-BC cases. This increased prevalence may be due to the high number of patients included who had received more than three treatment lines, since a higher $P I K 3 C A$ variant prevalence was described for heavily pretreated cohorts [41].

PIK3CA E726K has already been detected in BC tissue and was predicted to cause a gain of function as well as increased oncogenic transformation, which was also described for PIK3CA H1047R located in the activation loop of the kinase domain [42-44]. With regard to conferred mechanisms of resistance, sensitivity to everolimus was not affected by PIK3CA variants [45]. However, PIK3CA variants affected the PIK3/AKT pathway activation and the latter caused endocrine resistance [46]. Thus, besides the detected ESR 1 variants, the here described variants PIK3CA E726K and PIK3CA H1047R with dramatically increased AF under everolimus and exemestane in the serial cfDNA samples of one patient might mirror another resistance mechanism to exemestane.

\section{Variants of the ErbB protein family}

We found high prevalence of variants in the genes of the three ErbB protein family members $E R B B 2, E R B B 3$, and $E G F R$. This underlines the importance of $E G F R$ for BC growth and progression in HER2- BC cases [47]. Interestingly, however, ERBB2 P582L, ERBB2 P597L, and ERBB2 $\mathrm{P} 612 \mathrm{~L}$, all causing the change of proline to leucine, were found to be significantly associated with increased survival after diagnosis of metastasis. This finding questions the clinical conclusion of the genomic variants found in plasma, since the increased survival observed in $E R B B 2$ altered cases might be due to effective targeting of $E R B B 2$ altered tumor cells by therapy to result in detection of these genomic variants in the plasma [48]. 


\section{Variants of the BRCA protein family}

Alterations in proteins involved in the DNA repair pathways have a high impact on carcinogenesis. Individuals with germline $B R C A 2$ variants have an incidence of $57 \%-65 \%$ to develop BC [49]. BC patients with BRCA1 or BRCA2 mutations, however, were demonstrated to be sensitive to platinum agents and poly (ADP-ribose) polymerase (PARP) inhibitors [50]. Using deep sequencing of cfDNA, we here described a prevalence of $B R C A 1 / 2$ variants in $95 \% / 60 \%$ of the cohort. This high prevalence of $B R C A$ variants in cfDNA of HR+ HER2- MBC patients has never been described before, probably also due to limitations of coverage capacities [51-53]. It is of note that the mean AF of BRCA1/2 variants found in the cohort was $1.5 \% / 3.9 \%$, indicating the detection of somatic rather than germline variants.

\section{Prognostic value of cfDNA characteristics}

The accumulation of likely pathogenic and pathogenic variants might cause survival disadvantages for the patients, since we found a significant correlation of the number of pathogenic and likely pathogenic variants with survival after diagnosis of metastasis.

\section{Longitudinal monitoring across treatment}

The longitudinal cfDNA variant monitoring in one patient does not mirror the entire cohort, but was chosen as an example for the evolution of variants in the course of the disease, which might also be taken as an indicator of certain selective pressure that seems to happen during the observation time. The high AFs of PIK3CA and ESRI variants at progression under eribulin are consistent with the knowledge about the persistence of aromatase inhibitor-selected ESRI variants throughout subsequent therapies [2] and a general increase of variant AFs at therapy failure [54]. Due to the fact that all variants detected at the time point of progression under eribulin had already been detected at the previous sampling time points, the mechanism for acquired resistance to eribulin might not be caused by the observed variants themselves.

Interestingly, the appearance of ESRI and PIK3CA variants with AFs $>4 \%$ was already observed 8 months earlier than progression of the disease by visual staging. Thus, these variants appear more sensitive in monitoring the evolution and potential resistance than contemporary staging methods. The promising effect of cfDNA monitoring, in general, to predate treatment response changes by weeks to months was described before [11, 12].

In conclusion, comprehensive analysis of cfDNA variants by targeted deep sequencing in HR+ HER2- BC cases not only confirmed the benefit of UMIs in the variant verification, but also identified new promising variants with clinical relevance for monitoring, prognosis, and therapy stratification. More comparative research needs to be done, focusing on other BC subtypes and also on other BC relevant genes to further emphasize the advantages of deep sequencing of cfDNA for BC therapy management.

Acknowledgements We sincerely thank all patients for their participation in this research study. We highly valuate the dedication of Oliver Hoffmann for connecting clinical practice and research by diligent caring about the patient's medical needs and educating the patients about the presented research study. The authors highly appreciate the commitment of all involved nurses and physicians from the Department of Gynecology and Obstetrics, University Hospital of Essen, Germany for their assistance in sampling and educating the patients. Markus Storbeck rounded the sequencing analysis and always offered excellent advice. QIAamp MinElute ccfDNA Kits and QIAseq Targeted DNA Panel Kits were kindly provided by QIAGEN, Hilden, Germany. The sequencing analysis was funded by QIAGEN, Hilden, Germany.

\section{Compliance with ethical standards}

Conflict of interest CK, MT, PM, AKB, and RK declare that they have no competing interests. $\mathrm{KB}, \mathrm{SH}, \mathrm{MSH}$ and $\mathrm{PH}$ are/were employees at QIAGEN, Hilden, Germany. SKB is a consultant for QIAGEN, Hilden, Germany.

Open Access This article is distributed under the terms of the Creative Commons Attribution 4.0 International License (http://creativeco mmons.org/licenses/by/4.0/), which permits unrestricted use, distribution, and reproduction in any medium, provided you give appropriate credit to the original author(s) and the source, provide a link to the Creative Commons license, and indicate if changes were made.

\section{References}

1. Diaz LA, Bardelli A (2014) Liquid biopsies. Genotyping circulating tumor DNA. J Clin Oncol 32(6):579-586. https://doi. org/10.1200/jco.2012.45.2011

2. Schiavon G, Hrebien S, Garcia-Murillas I et al (2015) Analysis of ESR1 mutation in circulating tumor DNA demonstrates evolution during therapy for metastatic breast cancer. Sci Transl Med 7(313):313ra182. https://doi.org/10.1126/scitranslmed.aac7551

3. Butler TM, Johnson-Camacho K, Peto M et al (2015) Exome sequencing of cell-free DNA from metastatic cancer patients identifies clinically actionable mutations distinct from primary disease. PLoS One 10(8):e0136407. https://doi.org/10.1371/journ al.pone. 0136407

4. Heitzer E, Haque IS, Roberts CES et al (2019) Current and future perspectives of liquid biopsies in genomics-driven oncology. Nat Rev Genet 20(2):71-88. https://doi.org/10.1038/s4157 6-018-0071-5

5. Krawczyk N, Fehm T, Banys-Paluchowski M et al (2016) Liquid biopsy in metastasized breast cancer as basis for treatment decisions. Oncol Res Treat 39(3):112-116. https://doi. org/10.1159/000444605

6. Takeshita T, Yamamoto Y, Yamamoto-Ibusuki $\mathrm{M}$ et al (2017) Analysis of ESR1 and PIK3CA mutations in plasma 
cell-free DNA from ER-positive breast cancer patients. Oncotarget 8(32):52142-52155. https://doi.org/10.18632/oncotarget.18479

7. Dirican E, Akkiprik M, Özer A (2016) Mutation distributions and clinical correlations of PIK3CA gene mutations in breast cancer. Tumour Biol 37(6):7033-7045. https://doi.org/10.1007/s1327 7-016-4924-2

8. Fribbens C, O'Leary B, Kilburn L et al (2016) Plasma ESR1 mutations and the treatment of estrogen receptor-positive advanced breast cancer. J Clin Oncol 34(25):2961-2968. https:// doi.org/10.1200/JCO.2016.67.3061

9. Baselga J, Im S-A, Iwata H et al (2017) Buparlisib plus fulvestrant versus placebo plus fulvestrant in postmenopausal, hormone receptor-positive, HER2-negative, advanced breast cancer (BELLE-2). A randomised, double-blind, placebo-controlled, phase 3 trial. Lancet Oncol 18(7):904-916. https://doi. org/10.1016/s1470-2045(17)30376-5

10. Wan JCM, Massie C, Garcia-Corbacho J et al (2017) Liquid biopsies come of age. Towards implementation of circulating tumour DNA. Nat Rev Cancer 17(4):223-238. https://doi.org/10.1038/ nrc. 2017.7

11. Diehl F, Schmidt K, Choti MA et al (2008) Circulating mutant DNA to assess tumor dynamics. Nat Med 14(9):985-990. https:// doi.org/10.1038/nm.1789

12. Dawson S-J, Tsui DWY, Murtaza M et al (2013) Analysis of circulating tumor DNA to monitor metastatic breast cancer. N Engl J Med 368(13):1199-1209. https://doi.org/10.1056/NEJMoa1213 261

13. Oshiro C, Kagara N, Naoi Y et al (2015) PIK3CA mutations in serum DNA are predictive of recurrence in primary breast cancer patients. Breast Cancer Res Treat 150(2):299-307. https://doi. org/10.1007/s10549-015-3322-6

14. Xu C, Nezami Ranjbar MR, Wu Z et al (2017) Detecting very low allele fraction variants using targeted DNA sequencing and a novel molecular barcode-aware variant caller. BMC Genomics 18(1):5. https://doi.org/10.1186/s12864-016-3425-4

15. Bredemeier M, Kasimir-Bauer S, Kolberg H-C et al (2017) Comparison of the PI3KCA pathway in circulating tumor cells and corresponding tumor tissue of patients with metastatic breast cancer. Mol Med Rep. https://doi.org/10.3892/mmr.2017.6415

16. Keup C, Hahn P, Hauch S, Sprenger-Haussels M, Tewes M, Mach P, Bittner A-K, Kimmig R, Kasimir-Bauer S, Benyaa K (2018) Targeted PCR-based deep sequencing of cfDNA with unique molecular indices by a customized QIAseq Targeted DNA Panel. protocols.io. https://doi.org/10.17504/protocols.io.trfem3n

17. Meyer M, Stenzel U, Myles S et al (2007) Targeted high-throughput sequencing of tagged nucleic acid samples. Nucleic Acids Res 35(15):e97. https://doi.org/10.1093/nar/gkm566

18. Keup C, Storbeck M, Hauch S et al (2019) Cell-free DNA variant sequencing using CTC-depleted blood for comprehensive liquid biopsy testing in metastatic breast cancer. Cancers 11(2):238. https://doi.org/10.3390/cancers 11020238

19. Thompson JC, Yee SS, Troxel AB et al (2016) Detection of therapeutically targetable driver and resistance mutations in lung cancer patients by next-generation sequencing of cell-free circulating tumor DNA. Clin Cancer Res 22(23):5772-5782. https://doi. org/10.1158/1078-0432.CCR-16-1231

20. Pestrin M, Salvianti F, Galardi F et al (2015) Heterogeneity of PIK3CA mutational status at the single cell level in circulating tumor cells from metastatic breast cancer patients. Mol Oncol 9(4):749-757. https://doi.org/10.1016/j.molonc.2014.12.001

21. Gyanchandani R, Kvam E, Heller R et al (2018) Whole genome amplification of cell-free DNA enables detection of circulating tumor DNA mutations from fingerstick capillary blood. Sci Rep 8(1):17313. https://doi.org/10.1038/s41598-018-35470-9
22. Zill OA, Banks KC, Fairclough SR et al (2018) The landscape of actionable genomic alterations in cell-free circulating tumor DNA from 21,807 advanced cancer patients. Clin Cancer Res 24(15):3528-3538. https://doi.org/10.1158/1078-0432. CCR-17-3837

23. Meric-Bernstam F, Brusco L, Daniels M et al (2016) Incidental germline variants in 1000 advanced cancers on a prospective somatic genomic profiling protocol. Ann Oncol 27(5):795-800. https://doi.org/10.1093/annonc/mdw018

24. Grasso CS, Cani AK, Hovelson DH et al (2015) Integrative molecular profiling of routine clinical prostate cancer specimens. Ann Oncol 26(6):1110-1118. https://doi.org/10.1093/annonc/mdv134

25. Takeshita T, Yamamoto Y, Yamamoto-Ibusuki M et al (2018) ESR1 and PIK3CA mutational status in serum and plasma from metastatic breast cancer patients: a comparative study. Cancer Biomark Sect A Dis Markers 22(2):345-350. https://doi. org/10.3233/CBM-171161

26. Richards S, Aziz N, Bale S et al (2015) Standards and guidelines for the interpretation of sequence variants. A joint consensus recommendation of the American College of Medical Genetics and Genomics and the Association for Molecular Pathology. Genet Med 17(5):405-424. https://doi.org/10.1038/gim.2015.30

27. Kim N, Hong Y, Kwon D et al (2013) Somatic mutaome profile in human cancer tissues. Genomics Inform 11(4):239-244. https ://doi.org/10.5808/GI.2013.11.4.239

28. Tan H, Bao J, Zhou X (2015) Genome-wide mutational spectra analysis reveals significant cancer-specific heterogeneity. Sci Rep 5:12566. https://doi.org/10.1038/srep12566

29. Lawrence MS, Stojanov P, Polak P et al (2013) Mutational heterogeneity in cancer and the search for new cancer-associated genes. Nature 499(7457):214-218. https://doi.org/10.1038/nature12213

30. Kanwal M, Ding X-J, Song X et al (2018) MUC16 overexpression induced by gene mutations promotes lung cancer cell growth and invasion. Oncotarget 9(15):12226-12239. https://doi. org/10.18632/oncotarget.24203

31. Jeselsohn R, Yelensky R, Buchwalter G et al (2014) Emergence of constitutively active estrogen receptor-alpha mutations in pretreated advanced estrogen receptor-positive breast cancer. Clin Cancer Res 20(7):1757-1767. https://doi.org/10.1158/1078-0432. CCR-13-2332

32. Robinson DR, Wu Y-M, Vats $P$ et al (2013) Activating ESR1 mutations in hormone-resistant metastatic breast cancer. Nat Genet 45(12):1446-1451. https://doi.org/10.1038/ng.2823

33. Chu D, Paoletti C, Gersch C et al (2016) ESR1 mutations in circulating plasma tumor DNA from metastatic breast cancer patients. Clin Cancer Res 22(4):993-999. https://doi.org/10.1158/10780432.CCR-15-0943

34. Guttery DS, Page K, Hills A et al (2015) Noninvasive detection of activating estrogen receptor 1 (ESR1) mutations in estrogen receptor-positive metastatic breast cancer. Clin Chem 61(7):974-982. https://doi.org/10.1373/clinchem.2015.238717

35. Rahim B, O'Regan R (2017) AR Signaling in Breast Cancer. Cancers. https://doi.org/10.3390/cancers 9030021

36. Bergerat J-P, Céraline J (2009) Pleiotropic functional properties of androgen receptor mutants in prostate cancer. Hum Mutat 30(2):145-157. https://doi.org/10.1002/humu.20848

37. Chen C, Fang X, Sun S (2018) Diagnosis of polyglutamine spinocerebellar ataxias by polymerase chain reaction amplification and Sanger sequencing. Mol Med Rep 18(1):1037-1042. https:// doi.org/10.3892/mmr.2018.9043

38. Hickey TE, Irvine CM, Dvinge $\mathrm{H}$ et al (2015) Expression of androgen receptor splice variants in clinical breast cancers. Oncotarget 6(42):44728-44744. https://doi.org/10.18632/oncotarget .6296 
39. Antonarakis ES, Lu C, Luber B et al (2017) Clinical significance of androgen receptor splice variant-7 mRNA detection in circulating tumor cells of men with metastatic castration-resistant prostate cancer treated with first- and second-line abiraterone and enzalutamide. J Clin Oncol 35(19):2149-2156. https://doi.org/10.1200/ JCO.2016.70.1961

40. Cancer Genome Atlas Networks (2012) Comprehensive molecular portraits of human breast tumours. Nature 490(7418):61-70. https ://doi.org/10.1038/nature11412

41. Takeshita T, Yamamoto Y, Yamamoto-Ibusuki M et al (2018) Clinical significance of plasma cell-free DNA mutations in PIK3CA, AKT1, and ESR1 gene according to treatment lines in ER-positive breast cancer. Mol Cancer 17(1):67. https://doi. org/10.1186/s12943-018-0808-y

42. Chang MT, Asthana S, Gao SP et al (2016) Identifying recurrent mutations in cancer reveals widespread lineage diversity and mutational specificity. Nat Biotechnol 34(2):155-163. https://doi. org/10.1038/nbt.3391

43. Bader AG, Kang S, Vogt PK (2006) Cancer-specific mutations in PIK3CA are oncogenic in vivo. Proc Natl Acad Sci USA 103(5):1475-1479. https://doi.org/10.1073/pnas.0510857103

44. Gymnopoulos M, Elsliger M-A, Vogt PK (2007) Rare cancer-specific mutations in PIK3CA show gain of function. Proc Natl Acad Sci USA 104(13):5569-5574. https://doi.org/10.1073/pnas.07010 05104

45. Moynahan ME, Chen D, He W et al (2017) Correlation between PIK3CA mutations in cell-free DNA and everolimus efficacy in HR + , HER2- advanced breast cancer: results from BOLERO-2. Br J Cancer 116(6):726-730. https://doi.org/10.1038/bjc.2017.25

46. Loi S, Haibe-Kains B, Majjaj S et al (2010) PIK3CA mutations associated with gene signature of low mTORC1 signaling and better outcomes in estrogen receptor-positive breast cancer. Proc Natl Acad Sci USA 107(22):10208-10213. https://doi.org/10.1073/ pnas.0907011107

47. Masuda H, Zhang D, Bartholomeusz C et al (2012) Role of epidermal growth factor receptor in breast cancer. Breast
Cancer Res Treat 136(2):331-345. https://doi.org/10.1007/s1054 9-012-2289-9

48. Swystun LL, Mukherjee S, Liaw PC (2011) Breast cancer chemotherapy induces the release of cell-free DNA, a novel procoagulant stimulus. J Thromb Haemost 9(11):2313-2321. https://doi.org/10 $.1111 / \mathrm{j} .1538-7836.2011 .04465 . x$

49. Rebbeck TR, Friebel TM, Friedman E et al (2018) Mutational spectrum in a worldwide study of 29,700 families with BRCA1 or BRCA2 mutations. Hum Mutat. https://doi.org/10.1002/ humu. 23406

50. Lord CJ, Ashworth A (2017) PARP inhibitors: synthetic lethality in the clinic. Science (New York, N.Y.) 355(6330):1152-1158. https://doi.org/10.1126/science.aam7344

51. Peshkin BN, Alabek ML, Isaacs C (2010) BRCA1/2 mutations and triple negative breast cancers. Breast Dis 32(1-2):25-33. https ://doi.org/10.3233/BD-2010-0306

52. Weigelt B, Comino-Méndez I, de Bruijn I et al (2017) Diverse BRCA1 and BRCA2 reversion mutations in circulating cell-free DNA of therapy-resistant breast or ovarian cancer. Clin Cancer Res 23(21):6708-6720. https://doi.org/10.1158/1078-0432. CCR-17-0544

53. Hovelson DH, Liu C-J, Wang Y et al (2017) Rapid, ultra low coverage copy number profiling of cell-free DNA as a precision oncology screening strategy. Oncotarget 8(52):89848-89866. https://doi.org/10.18632/oncotarget.21163

54. Murtaza M, Dawson S-J, Tsui DWY et al (2013) Non-invasive analysis of acquired resistance to cancer therapy by sequencing of plasma DNA. Nature 497(7447):108-112. https://doi.org/10.1038/ nature 12065

Publisher's Note Springer Nature remains neutral with regard to jurisdictional claims in published maps and institutional affiliations. 